\title{
Complete photonic bandgaps in the visible range from spherical layer structures in dichromate gelatin emulsions
}

\author{
Jenny Hung, Mang Hin Kok, and Wing Yim Tam ${ }^{a}$ \\ Department of Physics and William Mong Institute of Nano Science and Technology, The Hong Kong \\ University of Science and Technology, Clear Water Bay, Kowloon, Hong Kong, \\ People's Republic of China
}

(Received 12 July 2008; accepted 13 December 2008; published online 6 January 2009)

\begin{abstract}
We have fabricated spherical layer structures that exhibit complete photonic bandgaps in the visible range in dichromate gelatin emulsions by holographic interference. The complete bandgap was not a result of the high dielectric contrast but was due to the fact that the spherical layer structure was isotropic with equal spacing in all accessible directions. Angular dependence spectral measurements of the spherical layer structures were in good agreement with the expected results from an ideal structure of dielectric concentric spherical shells with equal spacing. Our fabrication technique and results could pave the way for new applications using complete bandgap photonic crystals.
\end{abstract}

() 2009 American Institute of Physics. [DOI: 10.1063/1.3064165]

To achieve complete bandgap in photonic crystals in which electromagnetic wave propagation is prohibited in all directions, in analogy to the bandgaps for electrons in semiconductor devices, has always been the goal of many engineers and scientists ever since it was proposed a few decades ago. ${ }^{1}$ Usual dielectric photonic crystals such as the facecentered-cubic structure and the like exhibit only partial bandgaps in which electromagnetic wave propagation is prohibited only in specific directions. Photonic crystals exhibiting complete photonic bandgaps were proposed by Ho et $_{\mathrm{al}}{ }^{2}$ in the woodpile and diamond structures. However, despite the more favorable structural arrangements, these structures still require high dielectric contrast that is not easily available using ordinary dielectric materials, especially for the visible range. ${ }^{2-4}$ Attempts had been made to increase the dielectric contrast to achieve complete bandgap, but only limited to the microwave or infrared ranges. ${ }^{5}$ Quasicrystals having higher symmetry than periodic structures are possible candidates for achieving complete bandgaps in the visible range. ${ }^{6}$ Recent works using quasicrystals as photonic crystals are promising, but more studies are still needed in the visible range. ${ }^{7-9}$

Since spherical shell has the highest symmetry of all structures, it is expected that structures made of spherical shells are ideal for achieving complete bandgaps. Furthermore, concentric spherical shells can exhibit complete photonic bandgaps in any direction pointing toward the center of the shells. More importantly, they do not require high dielectric contrast materials, making them more favorable over other structures. It is a challenge to fabricate such a concentric spherical shell structure using the common microfabrication techniques. Among the various methods for fabricating photonic crystals, the multiphoton direct laser writing ${ }^{10}$ (DLW) and the holographic lithography ${ }^{11-13}$ (HL) techniques are attractive because they can be used to fabricate various structures. ${ }^{8-10,13}$ The HL method is easy to set up and thus has an advantage over the DLW method, which requires thestate-of-the-art facilities in addition to the time-consuming

\footnotetext{
a) Author to whom correspondence should be addressed. Electronic mail: phtam@ust.hk. Tel.: 852-2358-7490. FAX: 852-2358-1652.
}

serial processes. In the HL method the interference pattern from multiple coherent beams can be recorded in photoresists or photosensitive gelatin emulsions. In principle, concentric spherical pattern can be obtained by the interference between the light from a point source placed at the center of a spherical mirror and the reflected light from the spherical mirror. Based on this approach, we have fabricated spherical layer structures with various curvatures in dichromate gelatin (DCG) emulsions. The spherical layer structures behaved like curved mirrors and, more importantly, exhibited complete bandgaps in the visible range despite the low dielectric contrast of the DCG.

Figure 1(a) shows the experimental setup used to realize the point source-spherical mirror interference. A beam from an argon ion laser, with a $488 \mathrm{~nm}$ wavelength $(\lambda), 0.06-0.4$ $\mathrm{mW}$ of power, and $2.5 \mathrm{~mm}$ in diameter, was focused to a point by a $100 \times$ microscopic objective and reflected, after diverging from the focal point, by a spherical mirror placed at a distance equal to the radius of the curvature of the mirror from the focal point of the objective. The spherical mirror was obtained by coating the curved surface of a planoconvex lens with aluminum. The divergent beam and the reflected beam would then interfere to form a concentric spherical pattern that could be recorded as spherical layers with spacing given by $d=\lambda / 2 n$ (where $n$ is the refractive index of the DCG) by a DCG plate placed in between the objective and the mirror. The radius of curvature of the spherical layers $\rho$ could be varied by sandwiching the DCG plate between two glass spacers, with suitable thicknesses, in contact with the objective and the planoconvex lens, as shown in Fig. 1(a). The lens and the spacers were chosen to have the same refractive index as the substrate of the DCG plate, and index matching fluid was added to all interfaces to ensure good interference. After 1-50 s exposure the DCG plate was developed following the procedures as reported before and, since the DCG is sensitive to moisture, it was protected by wax sealing a $0.2 \mathrm{~mm}$ thick cover glass on top of the DCG emulsion. ${ }^{14}$ Samples of five different curvatures were fabricated by placing the DCG plate, $i=1-5$, at positions $d_{1, i}$ $=9.5,7.0,4.5,2.0$, and $1.0 \mathrm{~mm}$ from the focal point of the microscope objective, respectively. Note that $\rho_{i}$ can be ap- 


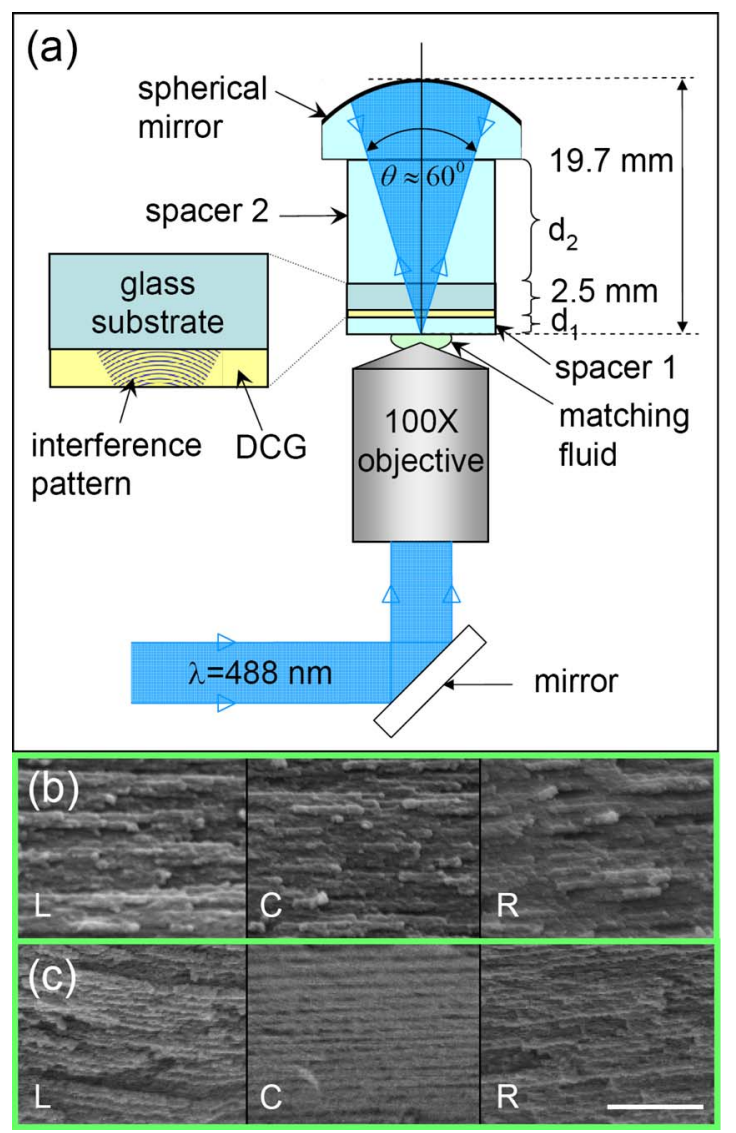

FIG. 1. (Color online) (a) Experimental setup for the fabrication of spherical layers using holographic interference. (b) Cross-section SEM images for plate 1 at positions: $300 \mu \mathrm{m}$ left (L) from center, center (C), and $400 \mu \mathrm{m}$ right $(\mathrm{R})$ from center of the sample. (c) SEM images for plate 4 at positions: $300 \mu \mathrm{m}$ left $(\mathrm{L})$ from center, center $(\mathrm{C})$, and $300 \mu \mathrm{m}$ right $(\mathrm{R})$ from center of the sample. The scale bar is $2 \mu \mathrm{m}$ for both (b) and (c).

proximated by $d_{1, i}$. Figures $1(\mathrm{~b})$ and $1(\mathrm{c})$ show scanning electron microscope (SEM) images of the spherical layer structures, left $(\mathrm{L})$, center $(\mathrm{C})$, and right $(\mathrm{R})$ of the sample, for plates 1 and 4, respectively. The curved nature of the layers is obvious despite some deformation of the gelatin emulsions caused by the developing and/or SEM preparation processes. Furthermore, the spacing of the spherical layers, $\sim 160 \mathrm{~nm}$ obtained from the SEM images of plate 1 in Fig. 1(b), agreed well with the expected result obtained by the point source-spherical mirror interference. Note that because of the limited divergence of the $100 \times$ objective, the spherical layers covered only about a $60^{\circ}$ conic section of a sphere.

The curved nature of the spherical layer structure is easily demonstrated by the reflection of an object from its surface. Figure 2(a) shows the setup for the reflection experiment. An object, a letter "F," was projected through an objective of a microscope onto the DCG sample with the spherical layers concaved upward. The reflections from the DCG samples are shown in Fig. 2(c) for plates 1-5. The reflected image, the letter $\mathrm{F}$ smaller than that of reflected from a plane mirror as shown in the inset of Fig. 2(a), resembled very much the reflection from a concave mirror. Furthermore, the reduction in the size of the image correlated well with the curvature of the spherical layers inside the DCG.

We used an optical microscope (Olympus BX60) coupled to a UV-visible spectrometer (Ocean Optics USB2000) through an optical fiber to measure the reflectance

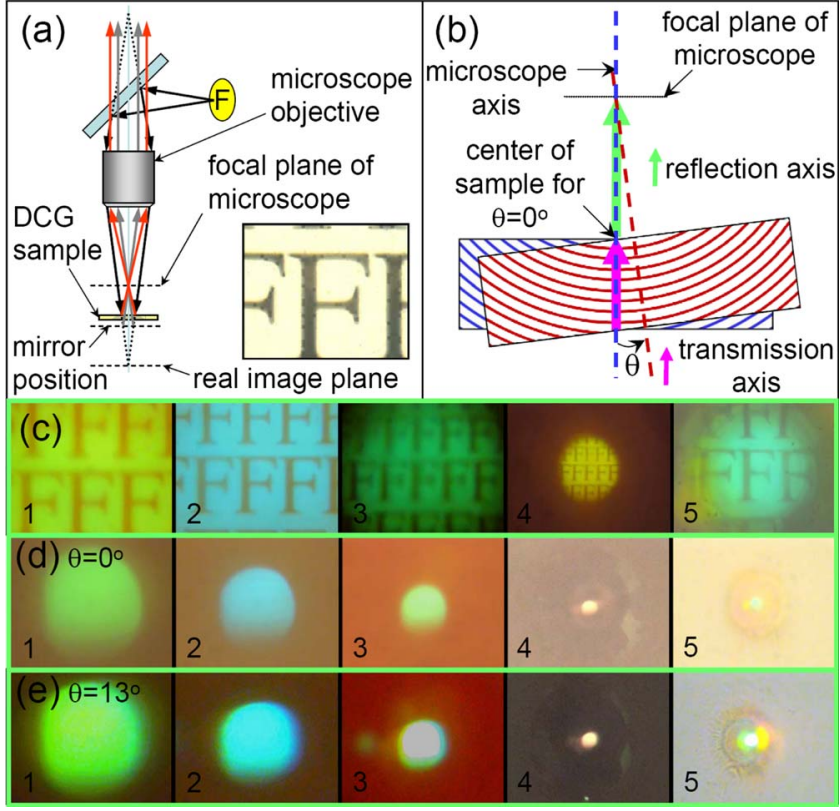

FIG. 2. (Color online) (a) Microscopic setup for reflection from the spherical layers in DCG samples. The inset is the reflection image of the object letter " $F$ " from a plane mirror using a $10 \times$ objective. (b) Configuration for angular dependence spectral measurements. (c) Reflection images of the object letter F from the spherical layer DCG samples using a $10 \times$ objective for plates 1-4 and a 50× objective for plate 5. [(d) and (e)] Focused images of the spherical layer DCG samples using a $5 \times$ objective for plates $1-4$ and a $10 \times$ objective for plate 5 at incident angle $\theta=0^{\circ}$ and $13^{\circ}$, respectively.

and transmittance of the spherical layer DCG samples. The microscope, with a small pin hole installed in the optical path, could sample a size down to $\sim 20 \mu \mathrm{m}$ in diameter using a $50 \times$ objective. Figures 3(a) and 3(b) show normal incidence transmission spectra at various distances from the center of the sample for plates 1 and 5, respectively. At the center of the sample, the transmission bandgap was the same as the one obtained from a planar structure. However, there was a blueshift of the transmission bandgap as the distance
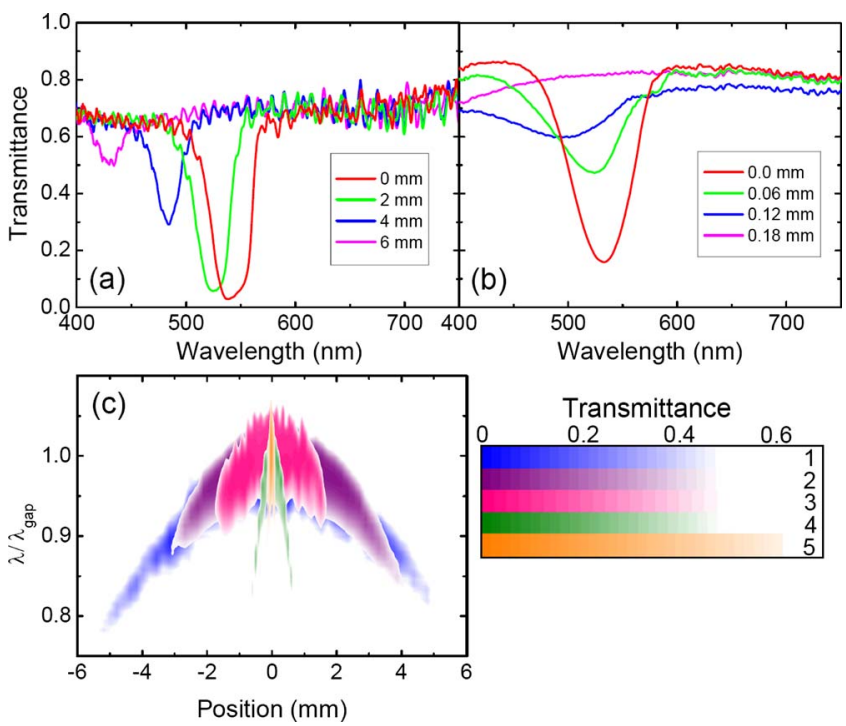

FIG. 3. (Color online) [(a) and (b)] Transmittance of the spherical layer DCG samples for plates 1 and 5, respectively, at different distances from the center of the samples. (c) Transmission spectra, shown as intensity contours with color bars on the right, along the diameter of the spherical layer DCG samples for the plates $1-5$. The wavelengths of the spectra are normalized to the midgap wavelengths $\lambda_{\text {gap }}$ of the bandgaps at the center of the sample. 


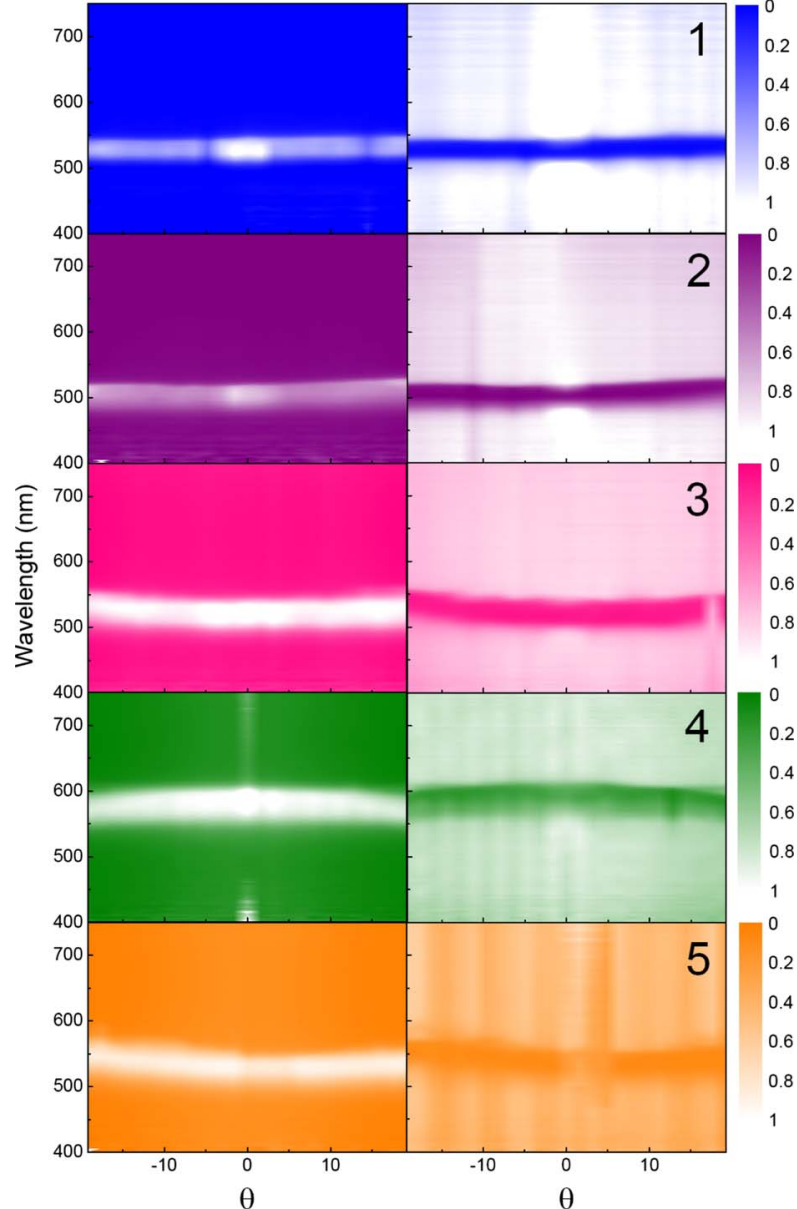

FIG. 4. (Color online) Angular reflectance (left column) and transmittance (right column) of the spherical layer DCG samples for plates 1-5.

from the center increased. This is consistent with the fact that moving away from the center of the spherical layer DCG sample is equivalent to incident at an angle on a planar structure, which will give a shorter wavelength bandgap compared to that of normal incidence. Figure 3(c) shows a superposition of the normal incidence transmission spectra scaled by the bandgap wavelengths at the center of the sample for plates $1-5$. The gradual blueshift with distance from the center and the decrease in the sample size with increasing curvature were obvious.

The spherical layer DCG samples exhibited a focusing effect just like a concave mirror, as shown in Figs. 2(d) and 2 (e). Under the microscope, the focused image, a bright circle with color at the bandgap of the spherical layer structure, also facilitated the alignment of the optical (reflection and transmission) axes of the sample with the microscope axis, as shown in Fig. 2(b). Figures 2(d) and 2(e) show the focused reflection images at normal and $13^{\circ}$ (measured inside the DCG gelatin) incidence, respectively. The color of the images remained unchanged at different incident angles, indicating that there was no change in the bandgap wavelengths. To better quantify the optical properties of the spherical layer DCG samples, we measured the angular reflectance and transmittance of the samples using the configuration in Fig. 2(b). Figure 4 shows the angular reflectance (left column) and transmittance (right column) over a range of incident angle from $-20^{\circ}$ to $20^{\circ}$ for plates $1-5$ as indicated. All spherical layer DCG samples showed flat reflectance and transmittance independent of the incident angle, a strong signature for complete bandgap. The same flat bandgaps were also observed over larger incident angles, from $-40^{\circ}$ to $40^{\circ}$, for plates $1-3$ using a rotation system for large samples as described before. ${ }^{9,15}$ Note that the peaks of the reflection bandgaps, white regions of the reflection bandgaps in the left column of Fig. 4, had reflectance larger than 1 because of the focusing effect of the curved layers. Figure 4 clearly confirmed the complete bandgaps for the spherical layer structures in DCG emulsions despite the low dielectric contrast of the gelatin.

To conclude, we have realized complete photonic bandgaps in the visible range from spherical layer structures in DCG emulsions fabricated using a simple holographic interference method. Despite the low refractive contrast of the DCG, robust complete bandgaps were obtained. Our samples covered only a small conic section of a sphere due to the limited divergence of the objective lens. Nevertheless, our results serve as a proof of principle that structures with concentric spherical shells are ideal photonic crystals to achieve complete bandgaps. Our technique and results could lead to new applications using complete bandgap photonic crystals.

Support from Hong Kong RGC grant (Grant Nos. HKUST602607 and HKUST602606) and a Central Allocation grant (Grant No. HKUST3/06C) are gratefully acknowledged.

${ }^{1}$ E. Yablonovitch, Phys. Rev. Lett. 58, 2059 (1987); S. John, ibid. 58, 2486 (1987).

${ }^{2}$ K. M. Ho, C. T. Chan, and C. M. Soukoulis, Phys. Rev. Lett. 65, 3152 (1990)

${ }^{3}$ C. T. Chan, S. Datta, K. M. Ho, and C. M. Soukoulis, Phys. Rev. B 50, 1988 (1994); M. Maldovan and E. L. Thomas, Nature Mater. 3, 593 (2004).

${ }^{4}$ T. Y. Chan, O. Toader, and S. John, Phys. Rev. E 71, 046605 (2005).

${ }^{5}$ N. Yamamoto, S. Noda, and A. Sasaki, Jpn. J. Appl. Phys., Part 1 36, 1907 (1997); A. Chutinan and S. Noda, Phys. Rev. B 57, R2006 (1998); S. Y. Lin, J. G. Fleming, D. L. Hetherington, B. K. Smith, R. Biswas, K. M. Ho, M. M. Sigalas, W. Zubrzycki, S. R. Kurtz, and J. Bur, Nature (London) 394, 251 (1998); A. Blanco, E. Chomski, S. Grabtchak, M. Ibisate, S. John, S. W. Leonard, C. Lopez, F. Meseguer, H. Miguez, J. P. Mondia, G. A. Ozin, O. Toader, and H. M. van Driel, ibid. 405, 437 (2000); Y. A. Vlasov, X. Z. Bo, J. C. Sturm, and D. J. Norris, ibid. 414, 289 (2001).

${ }^{6}$ Y. S. Chan, C. T. Chan, and Z. Y. Liu, Phys. Rev. Lett. 80, 956 (1998); X. Zhang, Z. Q. Zhang, and C. T. Chan, Phys. Rev. B 63, 081105 (2001).

${ }^{7}$ W. Man, M. Megens, P. J. Steinhardt, and P. M. Chaikin, Nature (London) 436, 993 (2005); A. Ledermann, L. Cademartiri, M. Hermatschweiler, C. Toninelli, G. A. Ozin, D. S. Wiersma, M. Wegener, and G. von Freymann, Nature Mater. 5, 942 (2006).

${ }^{8}$ W. Y. Tam, Appl. Phys. Lett. 89, 251111 (2006).

${ }^{9}$ J. Xu, R. Ma, X. Wang, and W. Y. Tam, Opt. Express 15, 4287 (2007).

${ }^{10}$ M. Deubel, G. von Freymann, M. Wegener, S. Pereira, K. Busch, and C. M. Soukoulis, Nature Mater. 3, 444 (2004); M. Deubel, M. Wegener, A. Kaso, and S. John, Appl. Phys. Lett. 85, 1895 (2004); S. Shoji, H. Sun, and S. Kawata, ibid. 83, 608 (2003).

${ }^{11}$ M. Campbell, D. N. Sharp, M. T. Harrison, R. G. Denning, and A. J. Turberfield, Nature (London) 404, 53 (2000); X. Wang, J. F. Xu, H. M Su, Z. H. Zeng, Y. L. Chen, H. Z. Wang, Y. K. Pang, and W. Y. Tam, Appl. Phys. Lett. 82, 2212 (2003); X. Wang, C. Y. Ng, W. Y. Tam, C. T. Chan, and P. Sheng, Adv. Mater. (Weinheim, Ger.) 15, 1526 (2003).

${ }^{12}$ D. C. Meisel, M. Wegener, and K. Busch, Phys. Rev. B 70, 165104 (2004); T. Y. M. Chan, O. Toader, and S. John, Phys. Rev. E 71, 046605 (2005)

${ }^{13}$ Y. K. Pang, J. C. W. Lee, H. F. Lee, W. Y. Tam, C. T. Chan, and P. Sheng, Opt. Express 13, 7615 (2005); Y. K. Pang, J. C. W. Lee, C. T. Ho, and W. Y. Tam, ibid. 14, 9013 (2006).

${ }^{14}$ R. Ma, J. Xu, and W. Y. Tam, Appl. Phys. Lett. 89, 081116 (2006).

${ }^{15}$ B. Jin, J. Xu, Y. K. Pang, W. Y. Tam, and J. Opt, Pure Appl. Opt. 10, 085204 (2008). 